\section{Paediatric oncology in Europe: current situation and future directions}

\section{R. Ladenstein}

St. Anna Children's Hospital and Children's Cancer Research Institute, Vienna, Austria

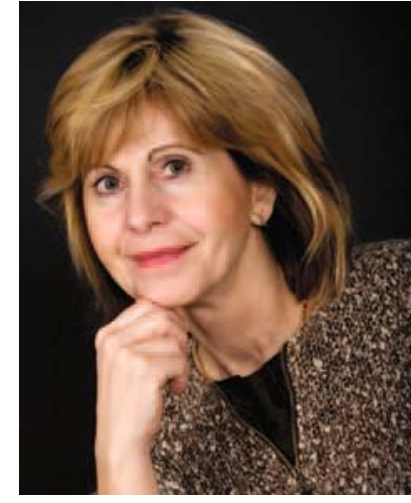

Assoc. Prof. Dr. Ruth Ladenstein
Paediatric Oncology is an orphan disease area needing the full support of the society to give children and adolescents an improved outlook for high quality long-term cure. Cancer in children is rare with approximately 1 in 500 affected and representing only $1 \%$ of all cancers in humans. It is very different from the typical epithelial cancers of adulthood.

Over the past 4 decades impressive improvement of overall cure rates was achieved from less than $20 \%$ to nearly $80 \%$ today. The basis was the outstanding collaborative work of national and international paediatric trial groups in investigator-driven clinical trials (IDCTs). However, cancer still remains the leading cause of death from disease beyond infancy in Europe and new innovative treatment approaches are needed.

Cancer in adolescents raises additional particular issues: delays in diagnosis, low participation in clinical trials as well as difficulties accessing the best standard of care for their tumour type and referral to clinical centres with the appropriate expertise. They are part of the patient group having benefited the least from progress in cancer treatment and their prognosis is, in general, particularly poor.

Almost 20,000 young people (aged up to $19 \mathrm{yrs}$ ) will be diagnosed with cancer this year in the EU. Although the majority can expect to be cured there are significant health problems in many survivors. This is a consequence of the long-term side effects of many current chemotherapy drugs and radiotherapy administered to the growing child. It is estimated that there are currently between 300,000 and 500,000 adults in Europe who have survived a cancer treated in their youth. This number is set to grow as survival rates improve.

Integration of research and care is a hallmark of paediatric oncology. More than $80 \%$ of children are treated either within clinical trials from phase I to phase III or within prospectively monitored therapeutic protocols that are applied in each paediatric malignancy and used by most paediatric oncology centres. Typical examples of paediatric IDCTs are proof-of-concept studies, novel indications for registered drugs and multi-modal therapy optimisation through strategic study planning over decades. In this way very successful standards of care for paediatric cancer were established, but

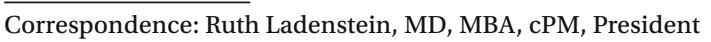
of SIOP-Europe, St. Anna Children's Hospital and Children's Cancer Research Institute, Kinderspitalgasse 6, 1090 Vienna, Austria. E-mail: ruth.ladenstein@stanna.at required much off-label use due to the lack of licensed indications for children with cancer. IDCT thus have a much broader scope and potential impact than industry-driven clinical trials. IDCTs form a key part of patient-oriented clinical research and create the basis to continually improve patient care. However, these studies deal with potential diagnostic and therapeutic innovations that do not attract commercial interest, particularly as paediatric cancer is an orphan disease area and thus there is very little funding from industry compared to the adult setting.

The variable implementation of the EU Clinical Trials Directive (EU CTD) into national law in 2004 caused additional problems for clinical trials in children. Many organisations (universities and hospitals or research organisations) are still unwilling to undertake the role of sponsor at a panEuropean level for multinational trials. In particular, the widely varying regulations and laws on liability between counties make sponsorship difficult to define. In addition, there is no consistent approach to the sponsorship of trials that are not investigating investigational medicinal products (IMPs). Especially, the high proportion of anti-cancer 'off-label' drugs used in the paediatric age group has led to highly variable definitions of investigational medicinal products (IMPs) across Europe. This has had major implications for the bureaucracy and expense of pharmacovigilance and insurance requirements and has provoked major difficulties due to the lack of financial resources.

Whilst the EU CTD aims to standardise the regulation and quality of trials, this has not yet been achieved in practice, whilst there has been no noticeable impact on the already excellent record for children's safety in research participation. To date, there is a lack of co-ordination and inevitably much duplication of the massive efforts and resources invested by the clinical trial groups to meet the requirements of the EU CTD. These hurdles need to be reduced by better harmonisation of procedures and a European-level dialogue with regulators and ethical approval boards to facilitate cancer care in children and adolescents.

At the same time, it appears that progress in improving survival rates using current conventional chemotherapy agents has reached its limits. The most refractory paediatric diseases with an urgent therapeutic need are high-risk leukaemias, metastatic bone and soft tissue sarcomas, high-risk neuroblastoma, malignant brain tumours, in particular brain stem tumours, and other metastatic tumour types which to- 
gether account for approximately one-third of all paediatric cancers and for $80 \%$ of deaths.

The challenge of the next decade will be to introduce the new generation of biologically targeted drugs to continue to make progress in improving survival rates and to improve the quality of cure of long-term survivors of childhood cancers. However, the access for children to innovative compounds developed for adults by pharmaceutical companies has been extremely poor in Europe in the last 20 years. Fortunately, the new regulatory framework in Europe has changed placing obligations and incentives on the pharmaceutical industry to develop paediatric investigation plans (PIPs) when an appropriate need is identified in the age group. Better preclinical model systems are therefore needed to test-proof the mechanism and develop appropriate biomarkers for use in clinical trials. New statistical methodologies and designs are needed to make the most efficient use of the relatively small numbers of children available for clinical trials, especially early phase trials. The recent EU Paediatric Medicine Regulation (entered into force on January 26th 2007, Regulation (EC) No $1901 / 2006$ ) is a major initiative in favour of the development of safe and effective medicines for children. It is crucial that this regulatory process meets the needs of children with cancer and is fully integrated into clinical and translational research to be run by or in partnership with the academic network.

The development of innovative therapies needs to be based on a better understanding of tumour biology, especially identifying the 'drivers' of malignant behaviour and the influence of interactions between tumour and host factors. Since biology matters, there is a need to support and fund basic and translational research as well as the full development of compounds, infrastructure of clinical research and network of clinical centres. A combination of European Union and national public funding as well as charitable donations is necessary to deliver the research agenda that will improve the cure rate and quality of cure of children and adolescents with cancer.

The European branch of the International Society of Paediatric Oncology (Siop E) fosters the ultimate goals to increase cure for children and adolescents with cancer, to improve quality of life during treatment and quality of survival and to improve access to the best standards of care and in- crease the capacity to deliver this standard throughout Europe. SIOP Europe has taken on the mission to meet the need to establish a durable, integrated clinical and translational research infrastructure for Europe that will define and implement its research strategy and will facilitate the necessary investigator-driven clinical trials to introduce the new generation of biologically targeted drugs into standard of care for children and adolescents with cancer. We are convinced that this will lead to more efficacious and less toxic therapies that will maximise the quality of life of the increasing number of survivors of cancer at a young age in Europe and allow them to assume their proper place in society. This biologically driven research agenda will improve the training methods of clinical investigators and translational scientists of the future to spread excellence and increase the capacity to participate in research and monitor outcomes across Europe. Patients and their families will be full partners and will be better informed about the need for and processes of clinical research. They will be in a better situation to care for the long-term health risks for children and potential outcomes. Drug development for children will be accelerated in partnership with industry through improved access to young patients with cancer, to academic expertise in care, clinical and biological research. All of this will be achieved with respect to the highest ethical and patient safety standards.

In this volume of MEMO two articles highlight, through the efforts of the EU-funded project "DIRECT", the previous EU activities to foster research projects in paediatric oncology and summarise nicely the current activities, as well as the needs and future aims of paediatric oncology.

Conflict of interest

The authors declare that there is no conflict of interest.

\section{References}

[1] Pritchard-Jones K; SIOP Europe. Clinical trials for children with cancer in Europe - still a long way from harmonisation: a report from SIOP Europe. Eur J Cancer, 44(15): 2106-2111, 2008. Epub 2008 Aug 29. Review.

[2] Vassal G. Will children with cancer benefit from the new European Paediatric Medicines Regulation? Eur J Cancer, 45(9): 1535-1546, 2009. 